\title{
A Direction-Based Location Update Scheme with a Line-Paging Strategy for PCS Networks
}

\author{
Hang-Wen Hwang, Ming-Feng Chang, and Chien-Chao Tseng
}

\begin{abstract}
On the problem of location update and terminal paging, many schemes using ring-paging strategies have been proposed. However, sequentially paging the rings surrounding the mobile user's last updated location may cause large paging cost. In this letter, we propose a direction-based location update (DBLU) scheme using a line-paging strategy to reduce the paging cost. A moving direction identification mechanism using only simple computations detects the change of moving direction and updates the mobile's location. The numerical results show that our DBLU scheme achieves good performance when the paging cost is high.
\end{abstract}

Index Terms-Line-paging, location update, ring-paging, terminal paging.

\section{INTRODUCTION}

$\mathbf{O}$ WING to the increasing population of mobile subscribers, smaller sized cells have been used to accommodate the large number of mobile terminals (MT's). Sending paging signals to all cells within a location area (LA) to locate an MT may result in an excessive amount of network bandwidth. Therefore, more sophisticated schemes [1]-[5] were proposed to make the location update and terminal paging operations more efficient. These schemes include the time-based, movement-based and distance-based schemes which locate an MT by paging the LA's ring by ring from its last updated location. In this paper, we propose a direction-based location update (DBLU) scheme where an MT updates its location only when its moving direction changes. To locate an MT, paging can be carried out along its moving direction, and hence the paging cost is reduced. Moreover. The MT's moving direction can be determined by simple numerical calculations.

\section{DiReCtion-BASEd Location UPDATE AND Line-PAging}

As shown in Fig. 1, we assume the network is homogenous and the LA's are hexagonal. An LA may consist of one or more cells. In the DBLU scheme, an MT performs a location update when its moving direction changes. In Fig. 1, for example, MT1 makes movements and its moving direction changes at points $\mathrm{B}, \mathrm{C}$, and D. After passing each direction-changing point, MT1 performs location update and informs the network of its new moving direction. Thus, the network can always keep track of

Manuscript received August 24, 1999. The associate editor coordinating the review of this letter and approving it for publication was Prof. N. B. Shroff.

The authors are with the Department of Computer Science and Information Engineering, National Chiao-Tung University, Hsinchu, Taiwan 300, R.O.C. (e-mail: hwhwang@csie.nctu.edu.tw; mfchang@csie.nctu.edu.tw; cctseng@csie.nctu.edu.tw).

Publisher Item Identifier S 1089-7798(00)03844-8.

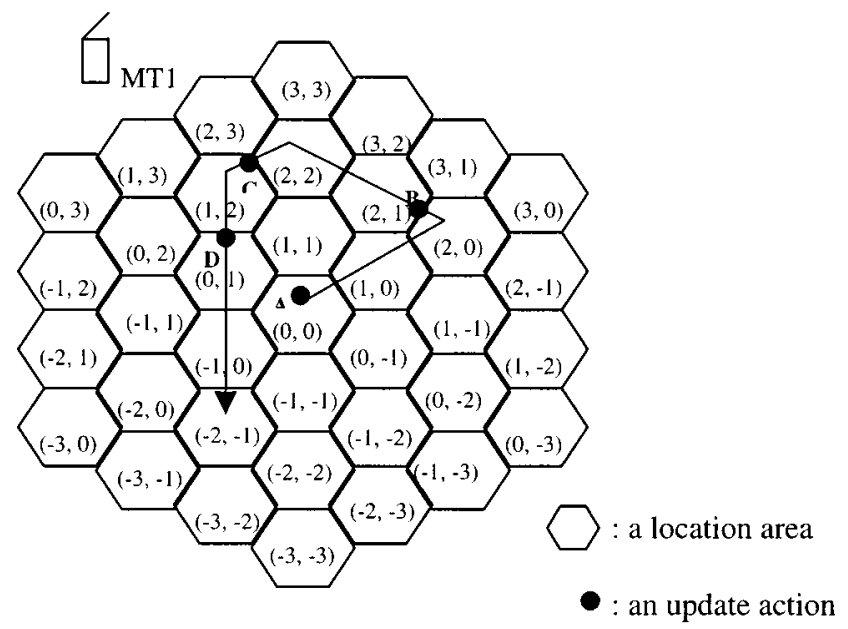

Fig. 1. Identification of location areas and an example moving MT.

MT1's moving direction. When a call is originated to a particular MT, the system only pages the LA's on the line of the MT's moving direction.

1) Direction Determination: As shown in Fig. 1, each LA can be identified with position coordinates $(X, Y)$. A sequence of LA's with position coordinates $\left(X_{1}, Y_{1}\right),\left(X_{2}, Y_{2}\right), \cdots$, and $\left(X_{n}, Y_{n}\right)$ are considered to be on a line if the location differences of every two adjacent LA's, $\left(X_{2}-X_{1}, Y_{2}-Y_{1}\right)$, $\left(X_{3}-X_{2}, Y_{3}-Y_{2}\right), \cdots$, and $\left(X_{n}-X_{n-1}, Y_{n}-Y_{n-1}\right)$, are the same. An MT is considered to keep the moving direction if it moves along LA's on a line. When an MT moves into a new LA, it computes the absolute values $\left(D X_{c}, D Y_{c}\right)$ of the location difference between the current location $\left(X_{i}, Y_{i}\right)$ broadcasted from the network, and the previous location $\left(X_{i-1}, Y_{i-1}\right)$ stored in the MT. Then, the MT compares the current location difference $\left(D X_{c}, D Y_{c}\right)$ with the previous location difference $\left(D X_{p}, D Y_{p}\right)$ stored in the MT. If $D X_{c} \neq D X_{p}$ or $D Y_{c} \neq D Y_{p}$, it means the MT has changed its moving direction. In this case, the MT should update its location and send the moving direction $\left(D X_{c}, D Y_{c}\right)$ to the network.

2) Line-Paging: When a call is originated to a particular MT, the ring-paging methods page all the adjacent LA's of the target MT ring by ring if the MT can not be found in the last updated LA. On the other hand, the DBLU scheme sequentially pages the LA's on the target MT's moving line until the MT is found. As the example shown in Fig. 1, if a call is originated to MT1 when it just moves to LA $(-2,-1)$, the DBLU scheme first pages the LA $(0,1)$, then pages LA $(-1,0)$ and $(1,2)$, and locates MT1 after paging LA $(-2,-1)$ and $(2,3)$. By contrast, 


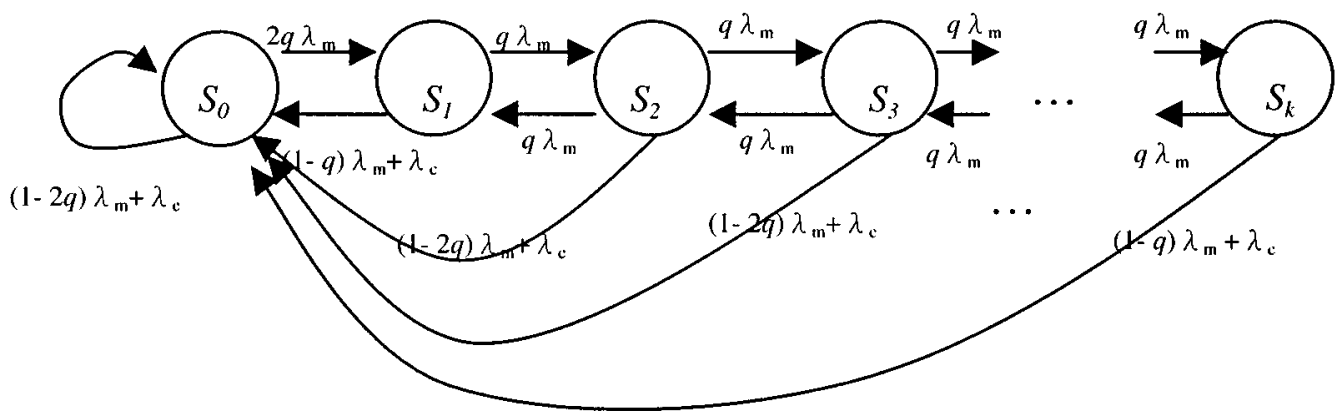

Fig. 2. State transition diagram for the DBLU scheme with a distance threshold $k$.

a ring-paging scheme may need to page 19 LA's [LA $(0,1)$ plus two rings] before MT1 can be located.

However, an MT may (even seldomly) fail to update the location when it changes its moving direction. In this case, the network can't locate the MT by the normal line-paging. Therefore, we adopt the concept of the distance-based scheme to limit the maximum distance that the MT can move on a line without making a new update. If a termination call arrives and the network fails in locating the MT by the normal line-paging scheme, the network will page the unpaged LA's ring by ring to locate the MT.

\section{PERformance AnAlysis}

We assume, for an MT, that the crossing LA movements and the calls to/from the MT are both Poisson processes with rates $\lambda_{m}$ and $\lambda_{c}$, respectively. Let us observe the MT only at each LA crossing and at each call arrival. We can model the activities of the MT by using an embedded Markov chain shown in Fig. 2. In the Markov chain, the system state $S_{i}$ represents that the distance between the MT's current LA and its last updated LA is $i$. We assume that an MT moves forward (from distance $i$ to distance $i+1$ ) or backward (from distance $i$ to distance $i-1$ ) in the same direction with equal probability $q$. Then, let $p_{i}$ denote the stationary probability of $S_{i}$, we can derive the following solutions from the balanced equations

$$
\begin{aligned}
\pi_{1} & =\frac{\left(1+\frac{\lambda_{c}}{\lambda_{m}}\right)\left(\pi_{0}-1\right)+2 q}{q}-\pi_{k} \\
\pi_{2} & =\frac{\left(1+\frac{\lambda_{c}}{\lambda_{m}}\right) \pi_{1}-2 q \pi_{0}}{q} \\
\pi_{i}= & \frac{\left(1+\frac{\lambda_{c}}{\lambda_{m}}\right) \pi_{i-1}-q \pi_{i-2}}{q}, \quad \text { for } k \geq i \geq 3 .
\end{aligned}
$$

We can obtain each $\pi_{i}$ by using numerical methods.

1) Total Cost: Let $C_{u}$ and $C_{p}$ denote the cost of one location update and one LA paging, respectively. Assume that each call generated for the MT is a termination call with probability $p_{t}$. At each LA crossing, an MT changes its moving direction with probability $1-2 q$. Besides, for a termination call to an MT at state $S_{i}$, the DBLU scheme needs to page $2 i+1$ LA's to locate the MT. Thus, the average total cost per unit time, $C$, can be expressed as

$$
\begin{array}{r}
C=\lambda_{m} C_{u}\left[(1-2 q) \sum_{i=0}^{k-1} \pi_{i}+(1-q) \pi_{k}\right] \\
+p_{t} \lambda_{c} C_{p} \sum_{i=0}^{k} \pi_{i}(2 i+1) .
\end{array}
$$

2) Random Walk Model: As shown in Fig. 1, each LA is surrounded by six LA's. In a random walk model, an MT moves to each adjacent LA with equal probability (1/6). Therefore, we can obtain the Markov chain for the random walk model by setting $q=1 / 6$. The average total cost per unit time can be expressed as

$$
C=\frac{2}{3} \lambda_{m} C_{u}+\frac{1}{6} \pi_{k} \lambda_{m} C_{u}+p_{t} \lambda_{c} C_{p} \sum_{i=0}^{k} \pi_{i}(2 i+1)
$$

3) An Enhancement: As mentioned before, the line-paging strategy significantly reduces the paging cost compared with the ring-paging one. However, if an MT changes its moving direction frequently, the cost of location update increases. To reduce the cost, we could plan the LA's on the path that most MT's pass, such as a highway or a main street, to form a preset line. As a result, the probability of an MT staying on the preset line may be larger than $1 / 3(2 q)$. We will demonstrate the enhancement by setting $q$ greater than $1 / 6$ in the enhancement model.

\section{NUMERICAL RESULTS}

To show the effectiveness of the DBLU scheme, we compare our scheme with the distance-based scheme [3]. Under a specific cost ratio $C_{p} / C_{u}$ and mobility-to-call ratio (MCR), namely, $\lambda_{m} / \lambda_{c}$, there exists an optimal distance threshold $d^{*}$ for the distance-based scheme to minimize the total cost. Fig. 3 shows the costs for the DBLU and distance-based schemes as the MCR varies from 0 to 10 . The DBLU scheme outperforms the distance-based scheme with the optimal distance threshold since the DBLU scheme significantly reduces the paging cost. However, under the random walk model, the DBLU scheme generates more location updates than the distance-based scheme with $d>0$. Thus, when the MCR is high $(\mathrm{MCR}>8)$ the location update cost becomes the dominating part, and the DBLU scheme performs worse than the distance-based scheme with the 


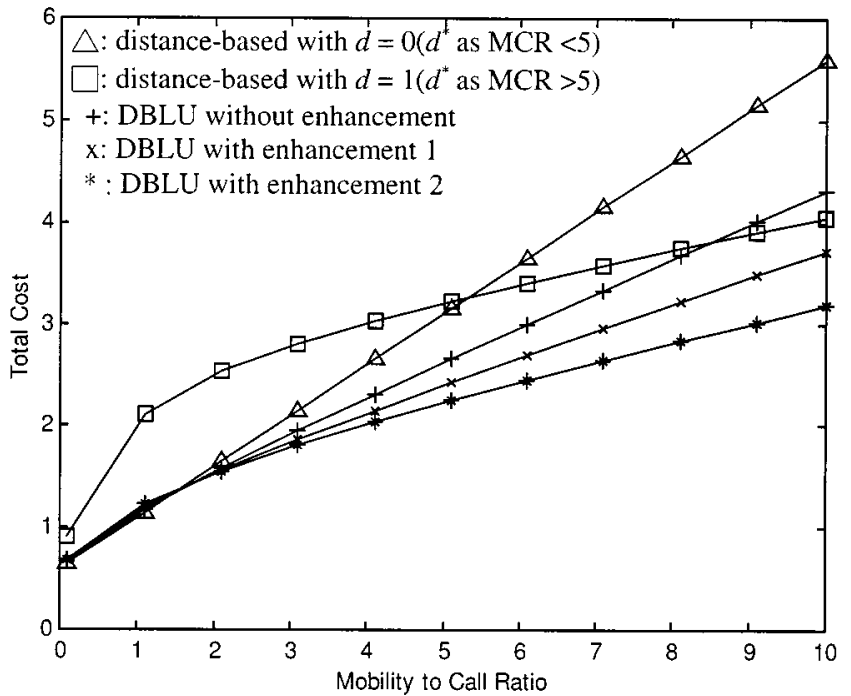

Fig. 3. Comparison of the total cost $\left(C_{u}=0.5, C_{p}=1, p_{t}=0.6 k=8\right.$ and $\lambda_{c}=1$ calls per unit time).

optimal distance threshold. As described previously, the DBLU scheme can be further improved by an enhancement based on the moving pattern of the majority of mobiles. In Fig. 3, the nu- merical results of enhancement case $1(q=1 / 4)$ and case 2 ( $q=1 / 3$ ) show that the update cost is significantly reduced.

\section{CONCLUSION}

In this letter, we propose a DBLU scheme with a line-paging strategy as an alternative location tracking method. The numerical results show that our scheme has good performance when the cost of paging one LA is comparable with that of one location update. Moreover, the DBLU scheme does not require complicated computations in both the MT and network. This merit makes the DBLU scheme feasible in location management.

\section{REFERENCES}

[1] A. Bar-Noy, I. Kessler, and M. Sidi, "Mobile users: To update or not to update?," Wireless Networks, vol. 1, no. 2, pp. 175-185, 1995.

[2] I. F. Akyildiz and J. S. M. Ho, "Dynamic mobile user location update for wireless PCS networks," ACM-Baltzer Wireless Networks, vol. 1, no. 2, pp. 187-196, 1995.

[3] J. S. M. Ho and I. F. Akyildiz, "Mobile user location update and paging under delay constraints," ACM-Baltzer Wireless Networks, vol. 1, no. 4, pp. 413-425, 1995.

[4] I. F. Akyildiz, J. S. M. Ho, and Y.-B. Lin, "Movement-based location update and selective paging for PCS networks," IEEE/ACM Trans. Networking, vol. 4, pp. 629-638, Aug. 1996.

[5] D. G. Jeong and W. S. Jeong, "Probabilistic location update for advanced cellular mobile networks," IEEE Commun. Lett., vol. 2, pp. 8-10, Jan. 1998. 